\title{
Calpain-1 expression is associated with relapse-free survival in breast cancer patients treated with trastuzumab following adjuvant chemotherapy
}

\author{
Sarah J. Storr ${ }^{1}$, Caroline M. Woolston ${ }^{1}$, Fabricio F.T. Barros ${ }^{2}$, Andrew R. Green ${ }^{2}$, Mohamed Shehata ${ }^{3}$, \\ Stephen Y. Chan ${ }^{3}$, Ian O. Ellis ${ }^{2}$ and Stewart G. Martin ${ }^{1}$ \\ Academic Oncology, University of Nottingham, School of Molecular Medical Sciences, Nottingham University Hospitals NHS Trust, City Hospital Campus, \\ Nottingham, United Kingdom \\ ${ }^{2}$ Histopathology, University of Nottingham, School of Molecular Medical Sciences, Nottingham University Hospitals NHS Trust, City Hospital Campus, \\ Nottingham, United Kingdom \\ Division of Clinical Oncology, Nottingham University Hospitals NHS Trust, City Hospital Campus, Nottingham, United Kingdom
}

The calpain family, and their endogenous inhibitor calpastatin, has been implicated in cancer progression, and recent in vitro data have indicated a role in trastuzumab resistance. The aims of our study were to examine expression levels of calpastatin, calpain-1 and calpain-2 in breast tumours from patients treated with trastuzumab following adjuvant chemotherapy to determine their potential as biomarkers to predict therapeutic response. The expression of calpastatin, calpain-1 and calpain2 was determined, using immunohistochemistry (IHC), in tumours from a series of 93 patients with primary breast cancer treated with surgery and adjuvant chemotherapy with or without trastuzumab followed by trastuzumab to complete 1 year of therapy. IHC was performed using tissue microarrays constructed from cores taken from intratumour and peripheral tumour areas. Expression was correlated with clinicopathologic variables and patient outcome. Calpastatin expression was correlated with Nottingham prognostic index $(p=0.003)$ and lymph node status $(p=0.007)$. Trastuzumab resistance was defined as disease relapse during therapy. Calpain-1 expression is associated with relapse-free survival $(p=0.001)$ and remained significant in multivariate analysis accounting for confounding pathological and treatment variables (hazard ratio 4.60, $95 \%$ confidence interval 1.05-20.25; $p=0.043$ ). Calpain-1 may be a useful biomarker to predict relapse-free survival in breast cancer patients treated with adjuvant trastuzumab and chemotherapy. A larger verification study is warranted.

HER2 belongs to the family of human epidermal growth factor receptors (EGFR, ErbB or HER), which has three other members: HER1 (EGFR), HER3 and HER4, and functions as receptor tyrosine kinases. Activation of the HER1, 3 and 4 occurs via a number of ligands promoting dimerisation. HER2 has no direct ligand and acts as a coreceptor by preferential formation of heterodimers with other family members. ${ }^{1}$ In breast cancer, a mutation leading to overexpression, or altered receptor structure, can cause the formation of heterodimer and homodimer, which result in overactivation and uncontrolled cellular growth (reviewed in Ref. ${ }^{2}$ ). Amplification of HER2 occurs in $15-30 \%$ of breast cancers and results in a more aggressive phenotype. ${ }^{3}$ Normal HER2 signalling

Key words: calpain, calpastatin, HER2, breast cancer, trastuzumab DOI: $10.1002 /$ ijc. 25832

History: Received 28 Sep 2010; Accepted 19 Nov 2010; Online 7 Dec 2010

Correspondence to: Dr. Stewart G. Martin, Academic Oncology, University of Nottingham, School of Molecular Medical Sciences, Nottingham University Hospitals NHS Trust, City Hospital Campus, Nottingham, NG5 1PB, United Kingdom,

Tel.: +44-(0)115-823-1846, Fax: +44-(0)115-823-1849,

E-mail: stewart.martin@nottingham.ac.uk can act via phosphorylation of phosphatidylinositol kinase-3 (PI3K) or through induction of Ras and affects cell survival and cell cycle progression. ${ }^{4}$ Trastuzumab (Herceptin) is a humanised anti-HER2 monoclonal antibody, approved for use in HER2-positive breast cancer that significantly improves disease-free survival after adjuvant chemotherapy. ${ }^{5-7}$ Trastuzumab has been postulated to exert antitumour effects via a number of potential mechanisms including antibodydependent cellular cytotoxicity, inhibition of signal transduction, proteolytic cleavage of HER2, angiogenesis and DNA repair. ${ }^{4}$ Trastuzumab improves the outcome for HER2-positive breast cancers; however, a number of patients can develop resistance to treatment. Certain tests have shown efficacy in predicting response to trastuzumab therapy in patients with metastatic cancer such as the measurement of serum levels of the extracellular domain of HER $2,{ }^{8}$ and there is evidence that low PTEN expression can predict resistance to trastuzumab in patients with metastatic disease treated with trastuzumab and taxane. ${ }^{9,10}$ However, PTEN expression does not reliably predict response in patients treated with trastuzumab containing neoadjuvant chemotherapy. ${ }^{11}$

The calpains and their endogenous inhibitor calpastatin have a role in a number of disease pathologies, notably carcinogenesis. The calpain family has been implicated in 
Table 1. Clinicopathologic variables of patient sample cohort

\begin{tabular}{ll}
\hline Variables & Number $(n=93)$ \\
\hline Age (years) & $54.88 \pm 9.57$ \\
\hline Tumour size $(\mathrm{mm})$ & $25.60 \pm 18.90$ \\
\hline Grade classification & \\
\hline I & $1(1.1 \%)$ \\
II & $22(23.7 \%)$ \\
\hline III & $67(72.0 \%)$ \\
\hline Unknown & $3(3.2 \%)$ \\
\hline Nottingham prognostic index & $5.05 \pm 0.96$ \\
\hline Lymph node status & \\
\hline Positive & $62(66.7 \%)$ \\
\hline Negative & $28(30.1 \%)$ \\
\hline Unknown & $3(3.2 \%)$ \\
\hline ER status & \\
\hline Negative & $51(54.8 \%)$ \\
\hline Positive & $42(45.2 \%)$ \\
\hline PR status & \\
\hline Low & $38(40.9 \%)$ \\
\hline High & $10(10.8 \%)$ \\
\hline Unknown & $45(48.4 \%)$ \\
\hline
\end{tabular}

Continuous data are shown as mean \pm standard deviation. Oestrogen receptor (ER) status was positive with an $\mathrm{H}$-score above 10 . Age had a minimum value of 30.7 and a maximum value of 74.8 years. Tumour size had a minimum of 2 and a maximum of $90 \mathrm{~mm}$. Nottingham prognostic index (NPI) had a minimum value of 2.28 and a maximum value of 7.28 .

various cellular processes including cell differentiation, transcriptional regulation, cytokine processing, migration and apoptosis. ${ }^{12,13}$ The calpains are calcium-dependent cysteine proteases including calpain-1 and -2 (EC3.4.22.52 and EC 3.4.22.53). Activation of calpain-2 can occur via the signalregulated kinase/mitogen-activated protein (ERK/MAPK) pathway, whereas calpain-1 can be activated by physiological calcium levels. ${ }^{14}$ Dephosphorylation by protein phosphatase 2A has been shown to reduce activity of calpain-1 and -2 in vitro. ${ }^{15}$ In cancer, calpain-2 has been implicated in the in vitro formation of invadopodia, which are associated with extracellular matrix degradation and increased invasive potential. $^{16}$

Calpain has been implicated in HER2 signalling pathways in a number of studies. Calpain-2 can be activated by EGFR through the ERK/MAPK pathway by direct phosphorylation of calpain. ${ }^{17,18}$ HER2 overexpression results in activation of the Akt and ERK pathways. The Akt pathway results in a number of events important in tumourigenesis such as signalling through the mTOR pathway. Importantly, HER2 has been shown to induce NFKB through the AKT pathway, which is maintained by calpain-mediated cleavage of the I $\mathrm{B}$ kinase complex. ${ }^{19}$ Recent evidence has indicated a further role for calpain in trastuzumab-resistant breast cancer. Kul- karni et al. investigated the influence of calpain-1 in trastuzumab-treated HER2-positive breast cancer in vitro. ${ }^{20}$ Their data suggested that calpain- 1 is activated following trastuzumab treatment and cleaves HER2 to disrupt signalling. In addition, they showed that trastuzumab-sensitive cells have higher calpain-1 activity than resistant cells; however, trastuzumab-resistant cells become dependent on calpain-1 activity for survival.

Interestingly, calpain is implicated in a number of interrelated pathways. For example, integrin engagement can cause focal adhesion kinase (FAK) phosphorylation, which results in ERK activation of calpain-1 to cleave FAK, which results in enhanced cell motility. ${ }^{21}$ FAK, like phosphatidylinositol $(3,4,5)$-triphosphase (PIP3), can be dephosphorylated by PTEN indicating pathway overlap.

The aims of our study were to $(i)$ analyse the expression pattern of calpastatin, calpain-1 and calpain-2 in HER2positive breast cancer and (ii) to determine if the expression of these proteins can predict relapse-free survival in HER2positive breast cancer patients treated with trastuzumab following adjuvant chemotherapy.

\section{Material and Methods Clinical samples}

Our study is reported in accordance with REMARK criteria. ${ }^{22}$ The expression of calpastatin, calpain-1 and calpain-2 was investigated using a tissue microarray (TMA) of 93 patients with primary breast cancer treated at Nottingham University Hospitals, King's Mill Hospital or Derby Hospitals between June 2004 and December 2009 with a median follow-up time of 30 months. Clinicopathologic variables of the cohort are recorded in Table 1. Patients were all treated with trastuzumab following surgery and chemotherapy. Trastuzumab treatment was given on a 3 weekly regimen for 52 weeks. Chemotherapy consisted of six cycles of 3 weekly fluorouracil $\left(500 \mathrm{mg} / \mathrm{m}^{2}\right)$, epirubicin $\left(100 \mathrm{mg} / \mathrm{m}^{2}\right)$ and cyclophosphamide $\left(500 \mathrm{mg} / \mathrm{m}^{2}\right)(\mathrm{FEC})$ combination $(n=53)$ or FEC-T consisting three cycles of FEC followed by three cycles of trastuzumab with concurrent docetaxel $\left(100 \mathrm{mg} / \mathrm{m}^{2}\right)$ per 3 weeks when patients were in a high-risk prognostic group according to local guidelines $(n=40)$. Patients with oestrogen receptor expression were offered adjuvant hormonal therapy $(n=40)$ following chemotherapy, and the majority of patients received adjuvant radiotherapy $(n=78)$. Treatment resistance was defined as relapse during therapy, and relapse-free survival was defined as the date from diagnosis to relapse with a mean relapse-free survival of 32 months; median survival for the group was not reached. Formalin-fixed paraffin-embedded tissue was used to create the TMA with one core per patient taken from intratumour and peripheral tumour areas. All patients had HER2 expression determined by IHC, and fluorescence in situ hybridisation was performed on cases with a score of $2+$ by IHC. At the end of the 
follow-up period, there had been one death, and 12 patients suffered a recurrence.

\section{TMA construction and immunohistochemistry}

Breast cancer TMAs were prepared. ${ }^{23} 0.6 \mathrm{~mm}$ tissue cores from both intratumour and peripheral tumour areas were placed into a single recipient paraffin block. Four-micron sections of the TMA were mounted on poly-L-lysine-coated slides. IHC was performed on the TMA slides, which were initially deparaffinised in histolene followed by rehydration in a series of ethanol baths (100, 90, 70, 50 and 30\% in water). Antigen retrieval was performed in $0.01 \mathrm{~mol} / \mathrm{L}$ sodium citrate buffer ( $\mathrm{pH}$ 6) in a microwave, $750 \mathrm{~W}$ for $10 \mathrm{~min}$ followed by $400 \mathrm{~W}$ for $10 \mathrm{~min}$. Endogenous peroxidase activity was blocked over $10 \mathrm{~min}$ in $0.01 \%$ hydrogen peroxide in methanol. Primary antibodies, mouse anti-calpastatin (1:20,000), mouse anti-calpain-1 (1:5,000) and rabbit anti-calpain-2 $(1: 5,000)$ (all Chemicon, MA), were diluted in blocking serum and applied to the tissue for $1 \mathrm{hr}$ at room temperature. Staining was achieved using the Vectastain Elite ABC kit (universal), containing blocking serum, biotinylated secondary antibody and $\mathrm{ABC}$ reagent (Vector Laboratories, Peterborough, UK). Immunohistochemical reactions were developed with 3,3'-diaminobenzidine as the chromogenic peroxidase substrate (Dako, Glostrup, Denmark). Sections were then counterstained with Gills formula Haematoxylin (Vector Laboratories), dehydrated and fixed in histolene before mounting with DPX. Breast tumour composite sections, which comprised of six Stage 1 breast tumours of Grade 1 to 3, were included as positive and negative controls with each run, with the negative control having primary antibody substituted for PBS.

Assessment of staining was conducted, after scanning of the slides with a Nanozoomer Digital Pathology Scanner (Hamamatsu Photonics), at $20 \times$ magnification. The expression of calpastatin, calpain-1 and calpain-2 in tumour cells was manually assessed using an immunohistochemical $H$-score. Staining intensity was assessed as: none (0), weak (1), medium (2) and strong (3) over the percentage area of each staining intensity. $H$-scores were calculated by multiplying the percentage area by the intensity grade ( $H$-score range: $0-300)$. Thirty percent of slides were examined by a second independent assessor blinded to scores and clinicopathologic criteria with good concordance between both scorers (single measure intraclass correlations greater than 0.8 ). An average $H$-score was generated by taking the mean of intratumour and peripheral tumour area cores and dichotomised using X-tile for analysis. ${ }^{24}$ Average scores were used for analysis because of the relatively small sample size.

\section{Statistical analysis}

The distribution of data was assessed using the KolmogorovSmirnov test for goodness of fit. The relationship between categorised protein expression and clinicopathologic factors was analysed using Pearson's Chi-square test of association $\left(\chi^{2}\right)$, where there were more than two variables e.g. grade, or Fisher's exact test in a $2 \times 2$ table. Overall disease-specific survival curves were plotted according to the Kaplan-Meier method, and significance was determined using the log-rank test. Multivariate survival analysis was performed by Cox proportional hazards analysis. Spearman rank order correlations were performed to test the associations between expression of proteins in intratumour and peripheral tumour locations, and between different proteins. All differences were deemed significant at the level of $p<0.05$. Statistical analysis was performed using SPSS 15.0 software.

\section{Results}

\section{Immunohistochemistry}

Tissue expression of calpastatin, calpain-1 and calpain-2 was determined in a series of trastuzumab-treated patients. Calpastatin, calpain-1 and calpain-2 demonstrated cytoplasmic staining with some granularity and heterogeneity between adjacent tumour cells, varying from weak to intense staining. Calpastatin had a median $H$-score of 130 and a standard deviation of \pm 55 ; calpain- 1 had a median value of $155 \pm 44$, and calpain- 2 core staining had a median value of $165 \pm 45$. Representative staining patterns from intracore and peripheral core are displayed in Figure 1.

A marginal biological correlation was observed between the tumour expression of calpastatin and calpain-2 $(r=0.231$, $p=0.027$ ), but not between calpastatin and calpain-1 or calpain-1 and calpain-2. Further relationships were explored by examining the relationship between localisation of expression in tumour cores. Significant correlation was observed between intratumour and peripheral tumour cores for calpastatin $(r=0.675, p<0.001)$ and calpain-1 $(r=0.391, p<$ $0.001)$ but not calpain-2 $(r=0.028, p=0.808)$.

\section{Relationship with clinicopathologic variables}

Calpastatin, calpain-1 and calpain- $2 H$-scores were dichotomised using $\mathrm{X}$-Tile software into low and high immunoreactivity and correlated with clinicopathologic criteria. X-tile-generated cut points were as follows: calpastatin had an $\mathrm{H}$-score cut point of 150 with 30 cases having a high score; calpain-2 had an $\mathrm{H}$-score cut point of 165 with 50 cases having a high score; calpain- 1 had an $H$-score of 215 with 10 cases having a high score. A small number of TMA cores were not assessed because of missing cores or insufficient representative tumour. Correlations with clinicopathologic criteria are shown in Table 2 for the three markers. Calpastatin expression correlated with Nottingham prognostic index (NPI) $(p=0.003)$ and lymph node status $(p=0.007)$. No significant correlations were observed with calpain-1 and calpain-2 expression.

The HER2 ratio (HER2/CEN-17) was determined for $78.5 \%$ (73/93) of cases using chromogenic in situ hybridisation, irrespective of IHC scoring value, and was tested for correlation with calpastatin, calpain-1 and calpain-2 expression using the Spearman rank correlation coefficient. Gene amplification ratios had a mean value of $3.6 \pm 0.8$. Calpastatin 
expression correlated with the HER2 gene amplification ratio $(r=0.258, p=0.027)$. No correlation was observed between calpain-1 or calpain-2 and HER2 gene amplification. HER2 IHC $H$-scores were also tested for correlation with calpastatin, calpain-1 and calpain-2 expression using the Spearman rank
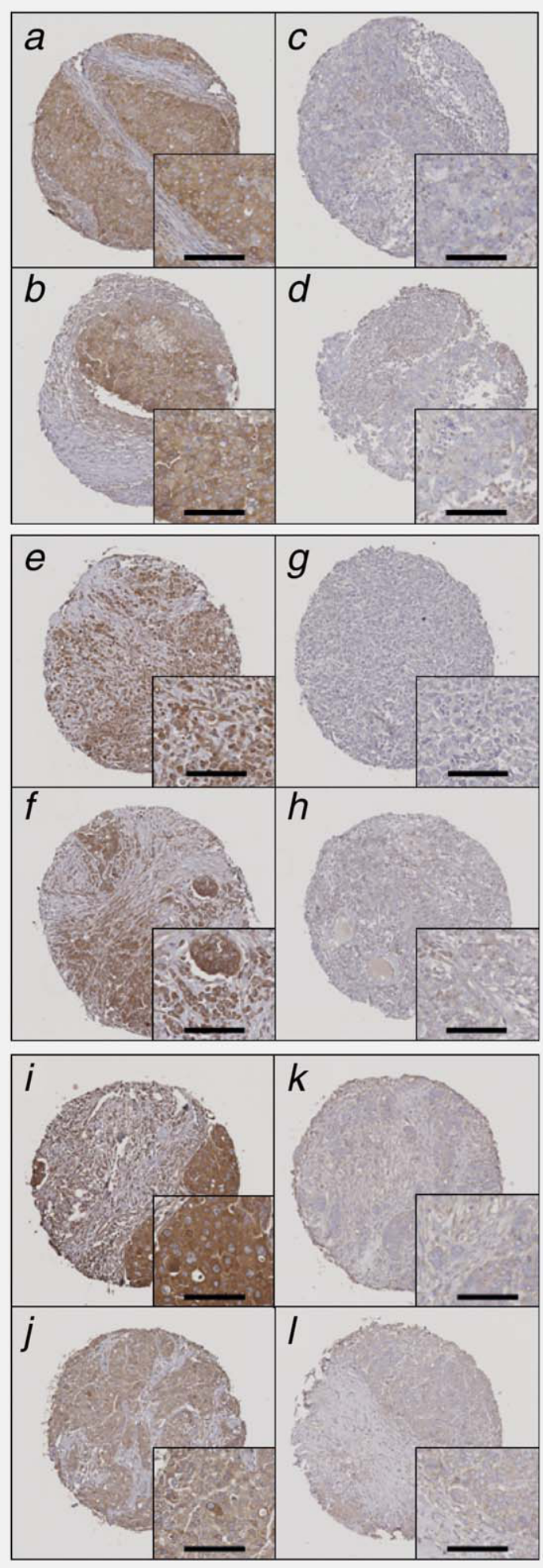

correlation coefficient. Calpastatin expression was correlated with the level of HER2 expression $(r=0.350, p=0.001)$, but no correlation was observed with calpain-1 and calpain-2.

\section{Relationship with clinical outcome}

Resistance to trastuzumab therapy was determined as patient relapse during therapy. The expression of calpastatin, calpain-1 and calpain-2 was correlated with relapse-free survival using Kaplan-Meier survival curves. The expression of calpain-1 was significantly associated with relapse-free survival $(p=0.001)$, with high expression indicating resistance to treatment (Fig. 2). No correlation was observed for calpastatin or calpain-2 expression and relapse-free survival (Fig. 2). Multivariate Cox regression analysis was performed including potential confounding factors such as tumour size, grade, lymph node status and ER status with individual Kaplan-Meier log-rank statistics of $p=$ $0.073, p=0.115, p=0.760$ and $p=0.062$, respectively. In addition, a cohort of other treatment factors was included such as type of surgery (wide local excision or mastectomy), adjuvant hormonal and radiotherapy, anthracycline or anthracycline and taxane chemotherapy and if chemotherapy was given in a neoadjuvant setting with individual Kaplan-Meier log-rank statistics of $p=0.060, p=0.054, p=0.086, p=0.600$ and $p=0.554$, respectively. Finally, a cohort including all possible confounding factors, both pathological and treatment bias, was included in multivariate Cox regression analysis. The expression of calpain-1 remained an independent marker for relapse-free survival with inclusion of pathologic confounders in the multivariate model (hazard ratio (HR) 4.265, 95\% confidence interval (CI) $1.22-$ $14.95 ; p=0.023$ ) but also of treatment setting (HR 4.434, 95\% CI $1.30-15.13 ; p=0.017$ ); finally, expression of calpain-1 was an independent marker of relapse-free survival including both pathologic and treatment confounding variables (HR 4.603, 95\% CI 1.05-20.25; $p=0.043$ ) (Table 3). No other markers were assessed by multivariate Cox regression as they did not correlate with relapse-free survival as single markers using Kaplan-Meier survival curves. No correlations with overall survival were possible as only one event occurred.

Simple tests of association using the Fisher's exact test were performed to assess the relationship between protein

Figure 1. Photomicrographs of representative staining at $\times 10$ magnification with $\times 20$ magnification inset panel with scale bar representing $100 \mu \mathrm{m}$. Intratumour (a) and peripheral (b) tumour high calpastatin staining from the same patient; intratumour (c) and peripheral (d) tumour low calpastatin staining from the same patient; intratumour $(e)$ and peripheral $(f)$ tumour high calpain-1 staining from the same patient; intratumour $(g)$ and peripheral (h) tumour low calpain-1 staining from the same patient; intratumour ( $i$ ) and peripheral ( $j$ ) tumour high and moderate calpain-2 staining from the same patient; intratumour $(k)$ and peripheral ( $l$ tumour low calpain-2 staining from the same patient. [Color figure can be viewed in the online issue, which is available at wileyonlinelibrary.com.] 
Table 2. Correlation between calpastatin, calpain-1 and calpain-2 protein expression and clinicopathologic variables

\begin{tabular}{|c|c|c|c|c|}
\hline \multirow[b]{2}{*}{ Variables } & \multirow[b]{2}{*}{$\begin{array}{l}\text { Number } \\
n=93\end{array}$} & \multicolumn{3}{|c|}{ Statistical $p$ value } \\
\hline & & $\begin{array}{l}\text { Calpastatin } \\
n=93\end{array}$ & $\begin{array}{l}\text { Calpain-1 } \\
n=93\end{array}$ & $\begin{array}{l}\text { Calpain-2 } \\
n=92\end{array}$ \\
\hline \multicolumn{5}{|l|}{ Age (years) } \\
\hline$\leq 40$ & $6(6.5 \%)$ & 0.383 & 1.000 & 0.684 \\
\hline$>40$ & 87 (93.5\%) & & & \\
\hline \multicolumn{5}{|c|}{ Tumour size $(\mathrm{cm})$} \\
\hline$\leq 2$ & $53(57.0 \%)$ & 0.178 & 0.512 & 0.524 \\
\hline$>2$ & 38 (40.9\%) & & & \\
\hline Unknown & $2(2.2 \%)$ & & & \\
\hline \multicolumn{5}{|c|}{ Grade classification } \\
\hline I & 1 (1.1\%) & $0.331^{\star}$ & $0.484^{\star}$ & $0.489^{*}$ \\
\hline ॥ & $22(23.7 \%)$ & & & \\
\hline III & $67(72.0 \%)$ & & & \\
\hline Unknown & $3(3.2 \%)$ & & & \\
\hline \multicolumn{5}{|c|}{ Nottingham prognostic index } \\
\hline Good & $3(3.2 \%)$ & $0.003^{*}$ & $0.711^{\star}$ & $0.245^{\star}$ \\
\hline Moderate & $49(52.7 \%)$ & & & \\
\hline Poor & $25(26.8 \%)$ & & & \\
\hline Unknown & $16(17.2 \%)$ & & & \\
\hline \multicolumn{5}{|c|}{ Lymph node status } \\
\hline Positive & $62(66.7 \%)$ & 0.007 & 1.000 & 0.359 \\
\hline Negative & 28 (30.1\%) & & & \\
\hline Unknown & $3(3.2 \%)$ & & & \\
\hline \multicolumn{5}{|l|}{ ER status } \\
\hline Negative & $51(54.8 \%)$ & 0.126 & 0.503 & 0.531 \\
\hline Positive & $42(45.2 \%)$ & & & \\
\hline \multicolumn{5}{|l|}{ PR status } \\
\hline Negative & 38 (40.9\%) & 0.727 & 1.000 & 0.724 \\
\hline Positive & 10 (10.8\%) & & & \\
\hline Unknown & 45 (48.4\%) & & & \\
\hline \multicolumn{5}{|c|}{ Relapse status } \\
\hline Relapse free & 81 (87.1\%) & 0.192 & 0.003 & 0.766 \\
\hline Relapse & 12 (12.9\%) & & & \\
\hline
\end{tabular}

Fisher's exact $(2 \times 2)$ test was used to determine significance, except where marked by * whereby the Pearson's Chi-square test of association was used.

Bold font indicates $p<0.05$

expression and relapse. Expression of calpain-1 was correlated with relapse status $(p=0.003)$ with $6.2 \%(5 / 81)$ of patients with low and $41.7 \%$ (5/12) of patients with high expression having relapsed disease. None of the other markers was correlated with relapse status (Table 2).

Interestingly, calpain-1 expression in the peripheral tumour core appears to be more informative for relapse-free survival than central tumour expression. By analysing each group using Kaplan-Meier survival curves, calpain-1 in the peritumoural area correlates with relapse-free survival $(p=$
$0.014)$, but not intratumoural staining $(p=0.247)$. It is unclear why a difference between the location of expression would play a role in treatment response.

\section{Discussion}

Our study investigated expression levels of calpastatin, calpain-1 and calpain-2 in 93 HER2-positive primary breast cancers treated with trastuzumab following adjuvant chemotherapy. The results show that expression of calpain-1 is able to predict relapse-free survival in patients treated with 
a

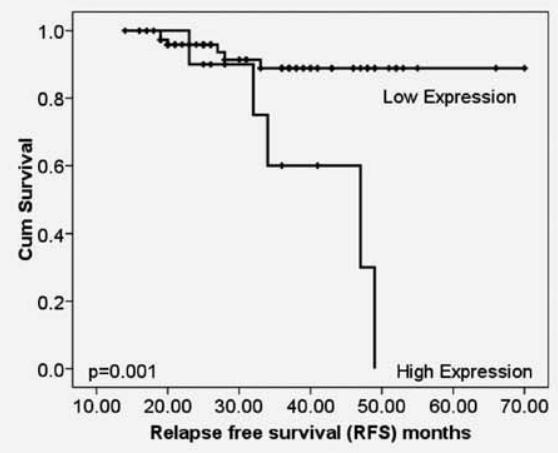

$b$

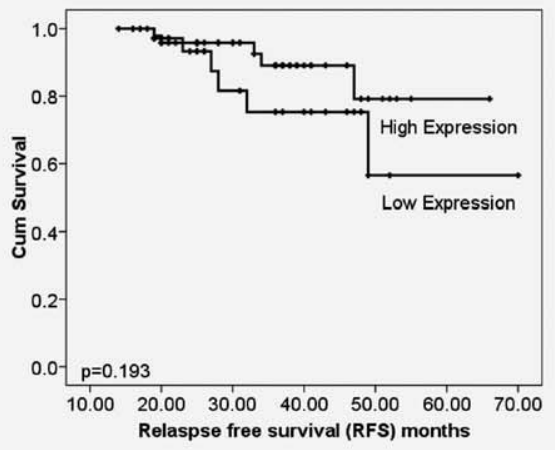

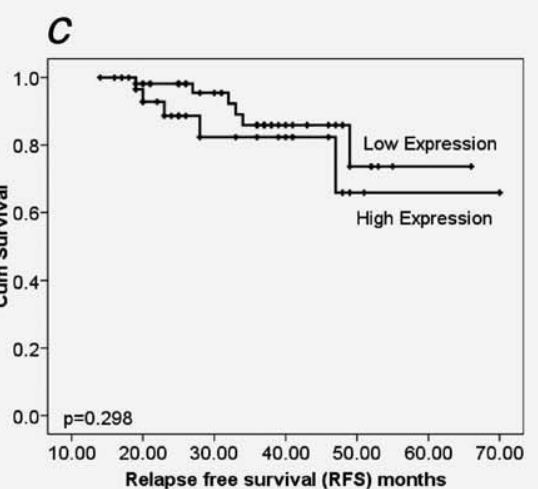

Figure 2. Analysis of relapse-free survival by Kaplan-Meier analysis of expression of calpain-1, calpain-2 and calpastatin determined by IHC. (a) Calpain-1 expression (83 patients low expression, 10 patients high expression); (b) calpain-2 expression (42 patients high expression, 50 patients low expression); (c) calpastatin expression (63 patients high expression, 30 patients low expression). Calpain-1 expression is significantly associated with relapse-free survival $(p=0.001)$, but calpain-2 and calpastatin are not.

Table 3. Multivariate Cox regression analysis showing calpain-1 expression, various pathological and clinical treatment variables and their effects upon relapse-free survival

\begin{tabular}{|c|c|c|c|c|c|c|c|c|}
\hline \multicolumn{9}{|l|}{ Multivariate Cox regression analysis } \\
\hline & \multicolumn{8}{|c|}{$95.0 \% \mathrm{Cl}$ for $\operatorname{Exp}(\mathrm{B})$} \\
\hline & $B$ & SE & Wald & df & Sig. & $\operatorname{Exp}(B)$ & Lower & Upper \\
\hline Calpain-1 expression & 1.527 & 0.756 & 4.081 & 1 & 0.043 & 4.603 & 1.046 & 20.245 \\
\hline Type of surgery & -0.613 & 0.767 & 0.639 & 1 & 0.424 & 0.542 & 0.120 & 2.435 \\
\hline Adjuvant radiotherapy & -7.514 & 11.358 & 0.438 & 1 & 0.508 & 0.001 & 0.000 & 2538707 \\
\hline Adjuvant hormonal therapy & 1.392 & 170.427 & 0.000 & 1 & 0.993 & 4.025 & 0.000 & $4.7 E+145$ \\
\hline Neoadjuvant/adjuvant chemotherapy & -1.356 & 1.227 & 1.221 & 1 & 0.269 & 0.258 & 0.023 & 2.856 \\
\hline Chemotherapy regimen & 0.829 & 0.812 & 1.041 & 1 & 0.308 & 2.291 & 0.466 & 11.256 \\
\hline Lymph node status & 5.796 & 11.334 & 0.262 & 1 & 0.609 & 329.057 & 0.000 & $1 E+012$ \\
\hline Oestrogen receptor status & -2.933 & 170.424 & 0.000 & 1 & 0.986 & 0.053 & 0.000 & $6.2 E+143$ \\
\hline Tumour grade & 10.042 & 18.386 & 0.298 & 1 & 0.585 & 22965.297 & 0.000 & $1 E+020$ \\
\hline Tumour size & 3.046 & 1.182 & 6.639 & 1 & 0.010 & 21.021 & 2.073 & 213.201 \\
\hline
\end{tabular}

The expression of calpain-1 and tumour size are independent predictors of trastuzumab relapse-free survival following adjuvant chemotherapy.

trastuzumab independent of pathological and clinical treatment variables $(p=0.043)$.

The findings presented in our study are interesting in the light of in vitro studies highlighting the role of calpain in HER2 signalling. ${ }^{19,20,25}$ The recent study by Kulkarni et al. implicated calpain-1 in resistance to trastuzumab treatment. ${ }^{20}$ Their results suggested that trastuzumab resistance is critically dependent on residual calpain activity through an impaired activation of calpain. It is difficult to draw comparisons between our study and previous in vitro work as our work examines calpain and calpastatin protein expression levels before treatment. The level of calpain activity in these specimens is unknown, although calpastatin expression may function to estimate this to some extent. In addition, the activity of calpain can be modulated by a number of factors such as physiological concentrations of calcium-activating calpain-1 and ERK/MAPK pathway activation of calpain-2. ${ }^{14}$ The data presented here show that high protein expression levels of calpain-1 are indicative of treatment outcome, with high expression levels correlating with a worse prognosis. Calpain activity has been implicated in the response to a number of therapeutic agents, such as cisplatin-induced apoptosis in lung adenocarcinoma cells through Bid activation, ${ }^{26}$ and has also been shown to degrade the DNA repair enzyme OGG1 in response to treatment. ${ }^{27}$ In addition, calpain-2 has been implicated in androgen receptor proteolysis to the constitutively active low-molecular-weight form in prostate cancer resulting through activation by ERK1 and $2 .{ }^{28}$

Calpain-1 expression was tested in multivariate analysis against other treatment variables; however, it is possible that calpain-1 expression may be influenced by these other treatment factors as well as trastuzumab alone. Calpain has been 
implicated in various tumourigenic processes such as altered adhesion and cellular migration. Previous work in our laboratory has established a link between calpastatin expression and lymphovascular invasion (LVI). This correlation was not made in our study as the data for IHC determination of LVI, as opposed to H\&E, were not available. ${ }^{29}$ In our study, calpastatin expression was significantly associated with NPI and lymph node status, with low levels of expression associated with more aggressive disease. Although calpastatin expression was associated with NPI, it was not associated with relapsefree survival; this was not unexpected as in our study NPI did not correlate with relapse-free survival $(p=0.132)$.

The expression of calpastatin correlates with calpain-2 expression, but not with calpain-1 expression. A correlation was not observed between calpain-1 and calpain-2 expression. It is unclear why a correlation is observed between calpain-2 and calpastatin, but not calpain-1; however, both of these enzymes have different activation requirements. In addition to the proteins investigated in our study, it would be interesting to investigate the activity of calpain. This might be possible using antibodies against calpain-specific degradation products; however, such reagents require further validation in human malignancies such as cancer. ${ }^{30,31}$ It would also be of interest to investigate other proteins that may be indicative of poor trastuzumab treatment response such as PTEN. ${ }^{9,11}$ A difference in expression was noted between calpain-1 intratumour and peripheral tumour cores used to determine the average $H$-score reported, with peripheral tumour staining more closely linked with relapse-free survival $(p=0.014$ and $p=$ 0.247 for peripheral tumour and intratumour, respectively). It is unclear what effect the tumour location of calpain-1 expression would play on response to treatment; however, the scores between cores taken from peripheral tumour and intratumoural areas had a statistically significant correlation $(p<0.001)$.

The definition of trastuzumab response in patients treated with combination therapy is challenging; however, the results of our preliminary study on a cohort of 93 patients present an exciting indication that calpain-1 expression levels are significantly associated with relapse-free survival in patients treated with trastuzumab following adjuvant chemotherapy. The median follow-up time in our study was 30 months; therefore, more patients in our cohort will be expected to relapse. ${ }^{32}$ Follow-up studies with larger patient cohorts with longer clinical follow-up are required, incorporating an investigation of calpain-1 expression and the response to other treatment modalities such as adjuvant chemotherapy alone. In particular, it must be noted that calpain appears to impart a more aggressive phenotype in various cancer models, including increased cellular motility. ${ }^{21}$ Although a small sample cohort, we have shown that expression of calpain-1 is significantly associated with relapse-free survival of patients treated with trastuzumab following adjuvant chemotherapy. Interestingly, the opportunity exists that antibodies that estimate the activity of calpain by the detection of specific degradation products, in combination with antibodies detecting calpain expression, may provide a useful tool in determining those patients who will not respond well to trastuzumab treatment following adjuvant chemotherapy. Calpain-1 may be a clinically relevant biomarker to determine HER2-positive patients who will relapse following trastuzumab therapy.

\section{Acknowledgement}

The authors gratefully acknowledge Cancer Research UK for funding S.J.S.

\section{References}

1. Olayioye MA, Neve RM, Lane HA, Hynes NE. The ErbB signalling network: receptor heterodimerisation in development and cancer. EMBO J 2000;19:3159-67.

2. Barros FFT, Powe DG, Ellis IO, Green AR. Understanding the HER family in breast cancer: interaction with ligands, dimerisation and treatments. Histopathology 2010;56:560-72.

3. Slamon DJ, Clark GM, Wong SG, Levin WJ, Ullrich A, McGuire WL. Human breast cancer: correlation of relapse and survival with amplification of the HER2/ neu oncogene. Science 1987;235:177-81.

4. Spector NL, Blackwell KL. Understanding the mechanisms behind trastuzumab therapy for human epidermal growth factor receptor-2 positive breast cancer. J Clin Oncol 2009;27:5838-47.

5. Piccart-Gebhart MJ, Procter M, LeylandJones B, et al. Trastuzumab after adjuvant chemotherapy in HER2-positive breast cancer. N Engl J Med 2005;353: 1659-72.
6. Smith I, Procter M, Gelber RD, et al. 2-Year follow up of trastuzumab after adjuvant chemotherapy in HER2-positive breast cancer: a randomised control trial. Lancet 2007;369:29-36.

7. Cho H-S, Mason K, Ramyar KX, Stanley AM, Gabelli SB, Denney DW, Leahy DJ. Structure of the extracellular region of HER2 alone and in complex with the Herceptin Fab. Nature 2003;421: 756-60.

8. Kostler WJ, Schwab B, Singer CF, et al. Monitoring of serum HER2/neu predicts response and progression free survival to trastuzumab-based treatments in patients with metastatic breast cancer. Clin Cancer Res 2004;10:1618-24.

9. Nagata Y, Lan K-H, Zhou X, Tan M, Esteva FJ, Sahin AA, Klos KS, Li P, Monia BP, Nguyen NT, Hortobagyi GN, Hung $\mathrm{M}-\mathrm{C}$, et al. PTEN activation contributes to tumour inhibition by trastuzumab and loss of PTEN predicts trastuzumab resistance in patients. Cancer Cell 2004;6:117-27.
10. Berns K, Horlings HM, Hennessy BT, Madiredjo M, Hijmans EM, Beelen K, Linn SC, Gonzalez-Angulo AM, Stemke-Hale K, Hauptmann M, Beijersbergen RL, Mills $\mathrm{GB}$, et al. A functional genetic approach identifies the PI3K pathway as a major determinant of trastuzumab resistance in breast cancer. Cancer Cell 2007;12:395-402.

11. Yonemori K, Tsuta K, Shimizu Y, Hatanaka Y, Hashizume K, Ono M, Kouno T, Ando M, Tamura K, Katsumata N, Hasegawa T, Kinoshita T, et al. Immunohistochemical expression of PTEN and phosphorylated Akt are not correlated with clinical outcome in breast cancer patients treated with trastuzumabcontaining neo-adjuvant chemotherapy. Med Oncol 2009;26:344-9.

12. Wendt A, Thompson VF, Goll DE. Interaction of calpastatin with calpain: a review. Biol Chem 2004;385:465-72.

13. Huang Y, Wang KK. The calpain family and human disease. Trends Mol Med 2001; $7: 355-62$. 
14. Glading A, Überall F, Keyse SM, Lauffenburger DA, Wells A. Membrane proximal ERK signalling is required for $\mathrm{m}$-calpain activation downstream of epidermal growth factor receptor signalling. J Biol Chem 2001;276:23341-8.

15. Xu L, Deng X. Suppression of cancer cell migration and invasion by protein phosphatase $2 \mathrm{~A}$ through dephosphorylation of $\mu$ - and m-calpains. J Biol Chem 2006; 281:35567-75.

16. Cortesio CL, Chan KT, Perrin BJ, Burton $\mathrm{N}$, Zhang S, Zhang Z-Y, Huttenlocher A. Calpain 2 and PTP1B function in a novel pathway with Src to regulate invadiopodia dynamics and breast cancer cell invasion. J Cell Biol 2008;180:957-71.

17. Glading A, Chang P, Lauffenburger DA, Wells A. Epidermal growth factor receptor activation of calpain is required for fibroblast motility and occurs via an ERK/ MAP kinase signalling pathway. J Biol Chem 2000;275:2390-6.

18. Glading A, Bodnar RJ, Reynolds IJ, Shiraha H, Satish L, Potter DA, Blair HC, Wells A. Epidermal growth factor activates $\mathrm{m}$ calpain (calpain II), at least in part, by extracellular signal related kinase mediated phosphorylation. Mol Cell Biol 2004;24: 2499-512.

19. Pianetti S, Arsura M, Romieu-Mourez R, Coffey RJ, Sonenshein GE. HER2/neu overexpression induces NFKB via a PI3kinase-Akt pathway involving calpain mediated degredation of I $\kappa \mathrm{B}-\alpha$ that can be inhibited by the tumour suppressor PTEN. Oncogene 2001;20:1287-99.

20. Kulkarni S, Reddy KB, Esteva FJ, Moore HCF, Budd GT, Tubbs RR. Calpain regulates sensitivity to trastuzumab and survival in HER2-positive breast cancer. Oncogene 2010;29:1339-50.

21. Sawhney RS, Cookson MM, Omar Y, Hauser J, Brattain MG. Integrin $\alpha 2-$ mediated ERK and calpain activation play a role in cell adhesion and motility via focal adhesion kinase signalling. J Biol Chem 2006;281:8497-510.

22. McShane LM, Altman DG, Sauerbrei W, Taube SE, Gion M, Clark GM. Reporting recommendations for tumour marker prognostic studies (REMARK). Nat Clin Pract Oncol 2005;2:416-22.

23. Abd El-Rehim DM, Ball G, Pinder SE, Rakha E, Paish C, Robertson JF, Macmillan D, Blamey RW, Ellis IO. High-throughput protein expression analysis using tissue microarray technology of a large well characterised series identifies biologically distinct classes of breast cancer confirming recent cDNA expression analyses. Int J Cancer 2005;116: 340-50.

24. Camp RL, Dolled-Filhart M, Rimm DL. X-Tile: a new bioinformatics tool for biomarker assessment and outcome based cut point optimisation. Clin Cancer Res 2004;10:7252-9.

25. Tan M, Li P, Klos KS, Lu J, Lan K-H, Nagata Y, Fang D, Jing T, Yu D. ErbB2 promotes Src synthesis and stability: novel mechanisms of Src activation that confer breast cancer metastasis. Cancer Res 2005; 65:1858-67.

26. Liu L, Xing D, Chen WR, Chen T, Pei Y, Gao X. Calpain mediated pathway dominates cisplatin induced apoptosis in human lung adenocarcinoma cells as determined by real time single cell analysis. Int J Cancer 2008;122: 2210-22.

27. Hill JW, Hu JJ, Evans MK. OGG1 is degraded by calpain following oxidative stress and cisplatin exposure. DNA Repair 2008;7:648-54.

28. Chen H, Libertini SJ, Wang Y, Kung H-J, Ghosh P, Mudryj M. ERK regulates calpain-2 induced androgen receptor in CWR22 relapsed prostate tumour cell lines. J Biol Chem 2010;285:2368-74.

29. Mohammed RAA, Martin SG, Gill MS, Green AR, Paish EC, Ellis IO. Improved methods for detecting lymphovascular invasion demonstrate it that is the predominant method of vascular invasion in breast cancer and has important clinical consequences. Am J Surg Pathol 2007;31: 1825-33.

30. Neumar RW, Meng FH, Mills AM, Xu YA, Zhang C, Welsh FA, Siman R. Calpain activity in rat brain after transient forebrain ischemia. Exp Neurol 2001;170: 27-35.

31. Gonçalves I, Nitulescu M, Saido TC, Dias N, Pedro LM, Fernandes e Fernandes J, Ares MPS, Pörn-Ares I. Activation of calpain-1 in human carotid artery atherosclerotic lesions. BMC Cardiovasc Disord 2009;9:26.

32. Romond EH, Perez EA, Bryant J, Suman VJ, Geyer CE, Davidson NE, Tan-Chiu E, Martino S, Paik S, Kaufman PA, Swain SM, Pisansky TM, et al. Trastuzumab plus adjuvant chemotherapy for operable HER2-positive breast cancer. N Engl J Med 2005;353: 1673-84. 